\title{
Three-dimensionality of the wake recovery behind a vertical axis turbine
}

\author{
Pablo Ouro*, Stefan Runge, Qianyu Luo, Thorsten Stoesser \\ Hydro-environmental Research Centre, School of Engineering, Cardiff University. The \\ Parade CF24 3AA, Cardiff, UK
}

\begin{abstract}
The wake recovery downstream of a vertical axis turbine operating in a turbulent channel flow is investigated via detailed velocity measurements using an Acoustic Doppler Velocimeter. Three distinct wake regions are identified: (i) a near-wake region which extends until two rotor diameters (2D) downstream and characterised by a low-momentum area isolated from the ambient flow and the presence of energetic dynamic stall vortices; (ii) a transition region (2D-5D), characterised by a fast momentum recovery, high turbulence intensity levels and vertical expansion of the wake; and (iii) a far-wake region beyond 5D where the velocity recovers to approximately $95 \%$ of the free-stream velocity. Albeit the wake deficit recovery is mostly accomplished at 5D behind the turbine, rotorinduced effects are still present beyond 10D as indicated by high-order flow statistics, such as high velocity fluctuations and flow skewness. Analysis of the streamwise momentum budget reveal that advection terms are mainly responsible for momentum replenishment through most of the wake and turbulent transport terms play only a minor role. This study evidences the anisotropic nature of the turbulence and asymmetry of the flow in horizontal, vertical and cross-sectional planes downstream of the vertical axis turbine.

Keywords: Vertical Axis Turbine, Wake Recovery, Array, Acoustic Doppler Velocimeter, Turbulence, Renewable Energy
\end{abstract}

\footnotetext{
${ }^{*}$ Corresponding author: OuroBarbaP@cardiff.ac.uk
} 


\section{Introduction}

With a growing demand for renewable and predictable energy, hydrokinetic energy from fast-flowing water bodies, such as ocean currents and river streams, could play a significant role in future energy strategies [1]. The design of hydroki-

5 netic turbines can be differentiated between horizontal axis turbines (HATs) and vertical axis turbines (VATs). To date, HATs are the most common choice in commercial tidal energy projects where they are believed to have reached a mature development in terms of efficient rotor design, due to their similarities to commercial wind turbines. On the other hand, investment in R\&D of VATs and their consequent application in tidal energy projects is limited despite their unique advantages such as high performance in shallow waters, omnidirectionality, low rotational speed and limited technical design requirements, which make them an attractive technology to a wide range of flows.

The flow regime experienced by VATs depends on the operating Reynolds number attained by the rotating blades, which will determine the relevance of dynamic stall of the blades or the mixing of the turbine's wake with the ambient flow. Bachant and Wosnik 2] tested a high-solidity VAT in a towing tank for different operational Reynolds numbers and found that drag forces and power coefficients tend asymptotically to a constant value, i.e. independent of the Reynolds number, for $R e_{D}=U D / \nu>1 \cdot 10^{6}$ or $R e_{c}>2 \cdot 10^{5}$. The acting forces on the blades are a consequence of the flow separation on their suction side which is fully turbulent once the former flow condition threshold is achieved. Using 2D-Partical Image Velocimetry (PIV), Simão-Ferreira [3] observed how flow separation on the blades varies depending on its rotational speed. Similarly,

25 Ouro and Stoesser [4] used high-fidelity numerical simulations to evidence the different nature of dynamic stall vortices whose effect becomes more prominent at low tip speed ratios.

The performance of different turbine designs in directly is also influenced by the generation of dynamic stall vortices and their convection within the rotor's swept perimeter which eventually interact back with the blades during the 
downstream half of the rotation. Brochier et al. [5] identified from water tank experiments how such blade-vortex interaction is characterised by leading- and trailing-edge vortices whose shedding pattern varies with the rotor's rotational speed. Somoano et al. [6] observed a varying wake recovery pattern at different tip speed ratios as a consequence of the vortex dynamics happening within the swept rotor area. With similar scope, Ouro and Stoesser [4] visualised in their large-eddy simulations that at low rotational speeds the blades undergo deep dynamic stall causing a drop in performance, whilst at high rotational speed, above peak performance, the blades lack of lift-generation capabilities to be sufficiently efficient. Therefore, an adequate balance in the interaction of the generated dynamic stall vortices with the moving blades is a key aspect in the development of efficient vertical axis turbines.

Early work on the three-dimensional nature of the wake behind vertical axis turbines was undertaken by Tescione et al. [7] who adopted PIV to measure different planes throughout the turbine's wake. Their phase-averaged results indicated the mechanism responsible for the generation of tip vortices as a result of the flow moving over the blade's tip and the low-pressure area on the blade suction side. These large-scale flow structures kept coherent until a distance of two turbine diameters (2D) downstream, over which a vertical expansion was also observed due to the action of tip vortices moving towards the outside of the turbine swept area. Thereafter these rotor-induced energetic structures merge with the low-momentum wake which starts to develop a vertical contraction.

In the mechanisms involved in the wake recovery in the near-wake, vertical advection of momentum appears to play a relevant role as discussed in Bachant and Wosnik [8]. Kinzel et al. 9] performed field measurements of different arrays of vertical axis wind turbines showing that vertical momentum transport is also of great significance in the wake recovery. It was further observed that rotor-induced turbulence aids in recovering wake momentum with wake velocity values close to the free-stream velocity at six diameters downstream, which suggests a closer cluster capability of VATs compared to HATs. Furthermore, Tescione et al. 7] also identified a circulation-induced phenomenon known as 
Magnus effect in the near-to-far wake transition which was also observed in the LES of Ouro and Stoesser [10] being this more noticeable at higher tip speed ratios.

Large-scale coherent motion of VATs' wakes were analysed by Araya et al. [11] for different turbine rotors equipped with 2 to 5 blades using spectral analysis and proper orthogonal decomposition. Three regions in a wake were observed: (i) near-wake, dominated by periodic blade vortex shedding; (ii) transitional region, where the shear-layer formed in between the turbine's lowvelocity wake and ambient flow became unstable; and (iii) far-wake, dominated by bluff body-like wake oscillations. The wake characteristics appeared to vary depending on the dynamic solidity, a parameter that correlates with the rotor's geometric solidity to the tip speed ratio. Low dynamic solidity values indicated a strong ability of the free-stream flow to penetrate through the rotor while high

75 values of this parameter caused a larger obstruction of the turbine rotor to the flow and thus enhancing bypass flows . Consequently, the larger amount of fluid flowing through the rotor the faster its wake recovers and vice versa.

Apart from dynamic stall as a key characteristic in the wake of vertical axis turbines, other key features in the wake of VATs were found by Tescione et 80 al. 7] who identified a skewed nature of the wake based on the time-averaged velocity distribution far downstream the device. Rolin et al. [12] employed a stereo-PIV technique and observed two pairs of asymmetrical-counter-rotating vortices at the horizontal edges of the wake as a result of a variation in cross-flow momentum. Analogously, Ryan et al. [13] observed that the strength of this ${ }_{85}$ pair of vortices is directly related to the turbine's rotational speed.

The present research provides a detailed insight into the three-dimensional dynamics of the wake developed behind a three-bladed Gorlov vertical axis turbine. Results include first-, second- and third-order flow statistics throughout the wake based on velocity measurements using ADV obtained from $0.75 \mathrm{D}$ to $9014 \mathrm{D}$ downstream of the turbine's rotor, quantification of dynamic stall vortices decay in the near-wake, and velocity deficit recovery in the streamwise direction. 


\section{Experimental setup}

\subsection{Laboratory configuration}

The experiments were conducted in a recirculating hydraulic flume at Cardiff

95 University which is $17 \mathrm{~m}$ long, $1.2 \mathrm{~m}$ wide and $1 \mathrm{~m}$ deep. The flow rate was controlled by an axial flow impeller with a constant value of $Q=0.75 \mathrm{~m}^{3} / \mathrm{s}$ providing a flow depth of $H=0.65 \mathrm{~m}$. A flowstraightener was installed at the inlet of the flume in order to smooth the approaching flow. A number of previous studies performed in this flume provided insights into the hydrodynamics of Savonious [14] and Darrieus [15] vertical axis turbines as well as horizontal axis turbines [16].

Velocity measurements of the flow in the flume without turbine (hereinafter free-stream flow conditions) were obtained at the proposed turbine position prior to its deployment. An Acoustic Doppler Velocimeter (ADV, Nortek Vectrino) was used to measure velocities in $\mathrm{x}, \mathrm{y}$ and $\mathrm{z}$ directions at 336 points over the selected cross-section. The measurements had an accuracy of $\pm 0.5 \%$ and were taken at a frequency of $200 \mathrm{~Hz}$ over a sampling period $\left(t_{n}\right)$ of $120 \mathrm{~s}$. Results were post-processed and filtered by removing those samples whose correlation coefficient values were inferior to $70 \%$ [17, and a signal-to-noise ratio (SNR) of $>15 \mathrm{~dB}$ was considered. The time-averaged velocity $U_{i}=(U, V, W)$ is calculated from the measured velocity field $u_{i}=(u, v, w)$ as,

$$
U_{i}=\frac{1}{t_{n}-t_{0}} \int_{t_{0}}^{t_{n}} u_{i}\left(t_{n}\right) d t
$$

Reynolds decomposition was applied to calculate the root-mean-square (rms) of the velocity fluctuation $u_{i}^{\prime}=\left(u^{\prime}, v^{\prime}, w^{\prime}\right)$ resulting from subtracting to the mean velocity $U_{i}$ the instantaneous velocity values $u_{i}$, i.e. $u_{i}^{\prime}=U_{i}-u_{i}$. The turbulence intensities $\sigma_{u_{i}}=\left(\sigma_{u}, \sigma_{v}, \sigma_{w}\right)$ are the root-mean-square (rms) of the velocity fluctuation $u_{i}^{\prime}$, and read,

$$
\sigma_{u_{i}}=\left(u_{i}^{\prime} u_{i}^{\prime}\right)^{0.5}
$$


The turbulent kinetic energy $(k)$ was calculated from the normal Reynolds stresses as,

$$
k=\frac{1}{2}\left(u^{\prime} u^{\prime}+v^{\prime} v^{\prime}+w^{\prime} w^{\prime}\right)
$$

Note that from hereinafter $u_{i}^{\prime}$ refers to its time-average value, and all flow variables are normalised with the free-stream velocity $U_{0}$ and the rotor diameter $D$.

\subsection{Free-stream flow conditions}

The approaching flow is depicted in Fig. 1 which presents vertical profiles of the mean streamwise velocity $\left(U / U_{0}\right)$ and turbulence intensities $\left(\sigma_{u_{i}} / U_{0}\right)$ in the centreline of the flume $(y / D=0.0)$ and contours of the streamwise velocity in a cross-section of the flume without turbine. The mean cross-sectional $U$ velocity was found to be $0.97 \mathrm{~m} / \mathrm{s}$ and this is considered the free-stream, or bulk velocity $U_{0}$. The bulk Reynolds $\left(R e_{H}=U_{0} H / \nu\right)$ and Froude $(F r=$ $\left.U_{0} /(g H)^{0.5}\right)$ numbers under these flow conditions were $R e_{H}=6.3 \cdot 10^{5}$ and $F r=0.38$, respectively. Note that due to ADV measurement limitations close to the channel bed, side-walls and water surface, flow velocities were measured between $-0.60<z / D<0.65$ in the vertical direction and $-1.55<y / D<1.55$ in the spanwise direction.

According to Fig. 19, the mean streamwise velocity fluctuation $\left(\sigma_{u} / U_{0}\right)$ was approximately equal to $15 \%$ at the centre of the channel whilst transversal $\left(\sigma_{v} / U_{0}\right)$ and vertical $\left(\sigma_{w} / U_{0}\right)$ turbulence intensities were $12.3 \%$ and $9.2 \%$, re120 spectively. The resulting anisotropic ratio $\sigma_{u}: \sigma_{v}: \sigma_{w}$ is 1.0:0.8:0.6 being slightly higher than those found in other channel flows, e.g. 1.0:0.71:0.55 [18, which is most likely caused by the high Reynolds number of the flow, the relatively narrow section of the channel and the flow asymmetry induced by the flume's impeller. Turbulence intensity values along the whole cross-section (not shown here) were fairly consistent with those found for the centreline shown in Fig. 17. 

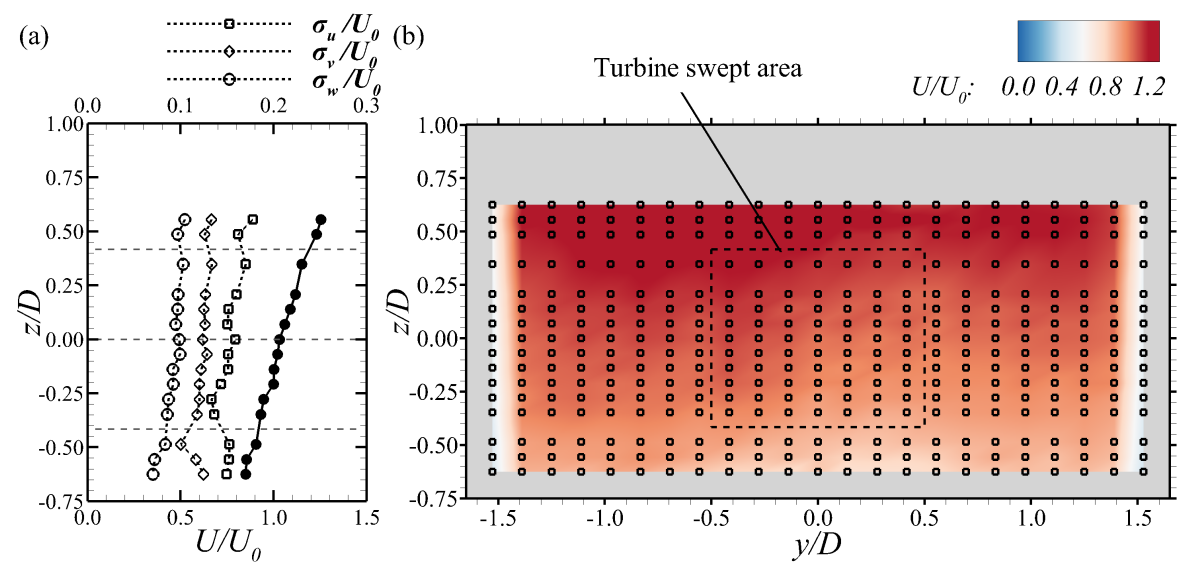

Figure 1: Data of the free-stream flow: (a) vertical profiles of mean streamwise velocity (solid line and filled symbols) and root-mean-square of velocity fluctuations (dashed lines and blank symbols); and (b) cross-section with contours of normalised time-averaged streamwise velocity.

\subsection{Description of the VAT}

A 1:10 scale three-bladed Gorlov-type VAT was placed in the previously described flume at $10 \mathrm{~m}$ downstream of the inlet. Blades and arms were made of laser-sintered PA 2200 material. Hub, shaft and submerged bearings were made of stainless steel. The rotor diameter $(D)$ was $0.36 \mathrm{~m}$ with a height $\left(H_{t}\right)$ of 0.30 $\mathrm{m}$ which gave a rotor aspect ratio $\left(D / H_{t}\right)$ equal to 0.85 . The blades featured a chord length $(c)$ of $0.08 \mathrm{~m}$ which resulted in a rotor solidity of $\sigma=21 \%$ which is calculated as,

$$
\sigma=\frac{N_{b} c}{\pi D}
$$

with $N_{b}$ denoting number of blades. The Reynolds number based on the diameter, $R e_{D}$, was approx. $3.5 \cdot 10^{5}$ and that based on the chord was $R e_{c}=7.8 \cdot 10^{4}$. Due to commercial sensitivity of the rotor design, no further geometry details can be given. The rotor swept cross-sectional area extended between $-0.5 D<y<0.5 D$ and $-0.4 D<z<0.45 D$ occupying $14 \%$ of the channel's cross-section.

The rotor was attached to a $0.02 \mathrm{~m}$ diameter shaft which drove a permanent magnet generator that converts the shaft's rotational motion into electrical 
energy and Fig. 2a depicts the turbine as deployed in the flume. Adjustable resistors were used to apply a resistance to the electrical circuit and dissipate energy in order to operate the rotor at the desired rotational speed. A combined encoder and torque sensor (Futek TRS605) was placed between the generator and rotor in order to measure rotational speed and torque. The rotor was held in place by a bearing adhered to the flume's bed, and another bearing that was attached to a bridge which rested on the side-walls of the flume well above the water surface (see Fig. 22p).
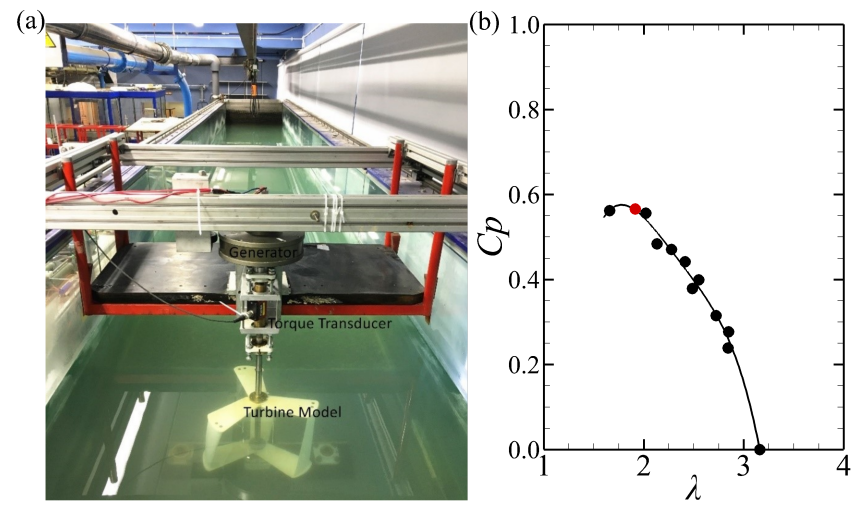

Figure 2: (a) Turbine rotor, generator and torque transducer assembled in the hydraulic flume at Cardiff University. (b) Measured power coefficient, $C_{p}$ versus tip speed ratio, $\lambda$, curve with the point of maximum efficiency indicated by a red symbol.

The performance of the turbine was calculated from the applied torque $(T)$ and rotational speed $(\Omega)$ monitored over a period of $60 \mathrm{~s}$, starting from freewheeling conditions (no torque applied / open electrical circuit) and progressively incrementing the applied torque (increased electrical resistance) until the rotor stalled, i.e. stopped rotating. The turbine efficiency was measured in terms of power coefficient, $C_{p}$, defined as,

$$
C_{p}=\frac{T \Omega}{\frac{1}{2} \rho A U_{0}^{3}}
$$

where $\rho$ is the density of water and $A=H_{t} D$ is the rotor's swept area. The performance curve is presented in Fig. $2 \mathrm{~b}$ in which rotational speeds are normalised by the water velocity and represented by the tip speed ratio $\lambda$, defined 
as,

$$
\lambda=\frac{\Omega D}{2 U_{0}}
$$

The highest efficiency was attained at $\lambda=1.9$ with a peak $C_{p}$ value of $y / D=0$

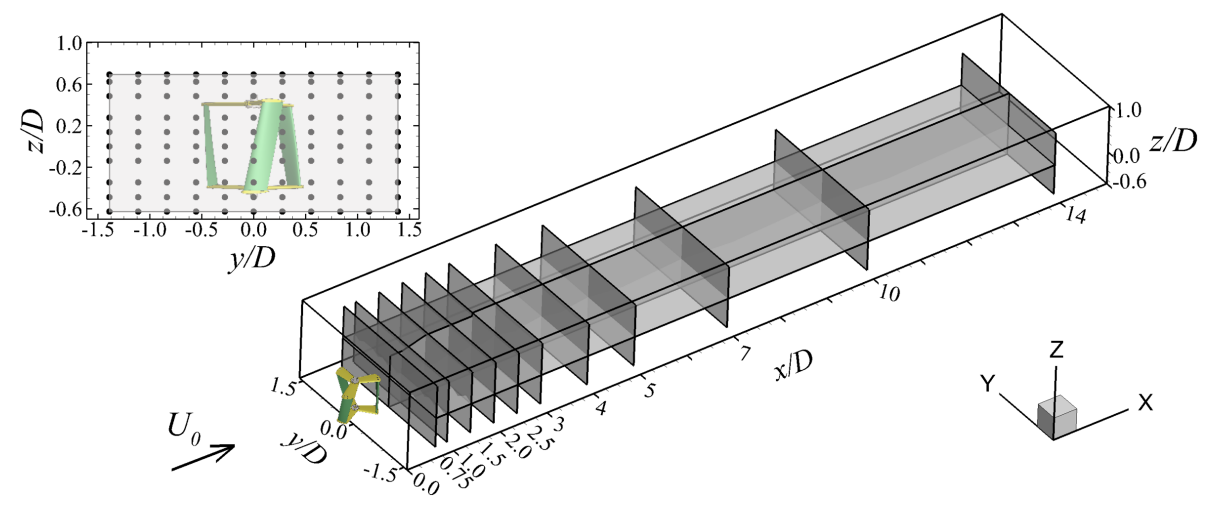

Figure 3: Location of the cross-sections behind the vertical axis turbine along the hydraulic flume and including velocity measurement points in the cross-section (top left insert). 


\subsection{Horizontal plane at mid-height of the turbine rotor}

Figure 4 presents an overview of the downstream evolution of the wake behind the turbine with the distribution of mean streamwise velocity $\left(U / U_{0}\right)$,

is different. Note that at $x / D=0.0, y / D=0.5$ the blades are moving into the flow experiencing the maximum relative velocity, whereas at $x / D=0.0$, 

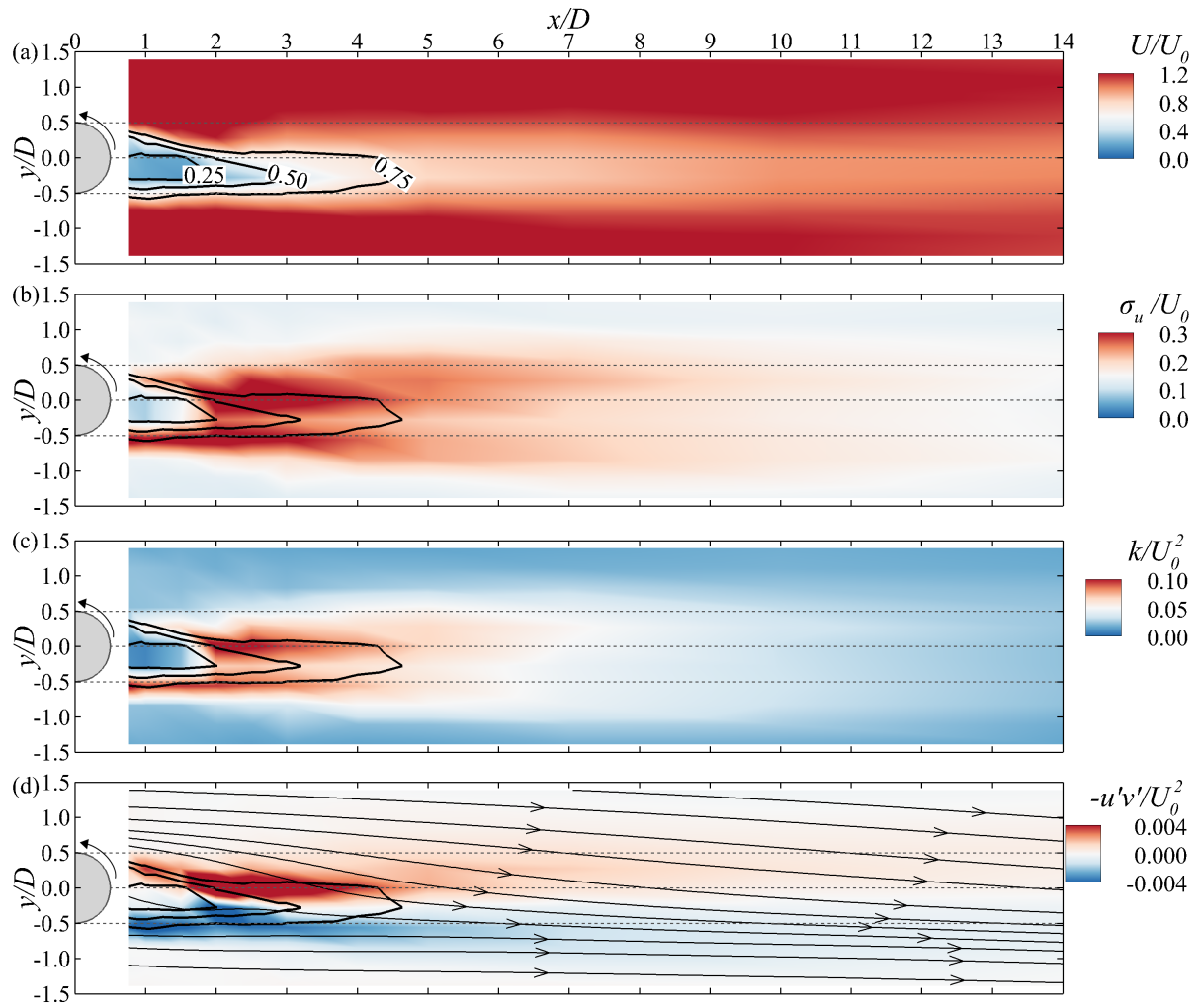

Figure 4: Horizontal plane at $z / D=0$ with distribution of mean (a) streamwise velocity, (b) streamwise velocity fluctuation, (c) turbulent kinetic energy, and (d) Reynolds shear stress $-u^{\prime} v^{\prime}$. Solid line indicates $U=0.25 U_{0}$.

$y / D=-0.5$ the blades move with the flow, i.e. attaining the minimum relative velocity. The rotor's rotation is responsible for the wake asymmetry in the horizontal plane.

Figure 5 presents horizontal profiles of streamwise mean velocity (a) and velocity fluctuation (b), Reynolds shear stress $-u^{\prime} v^{\prime}$ (c) and streamwise velocity gradient (d) at mid rotor height and at selected locations downstream of the turbine rotor. These plots allow to quantify the asymmetry of the flow and its turbulence features. Minimum values of $U$ are found predominantly over the $y / D<0$ side and the asymmetry is more visible closer to the turbine rotor. Note that the bypass flow, i.e. at $|y / D|>0.5$, features streamwise velocities of approximately $U / U_{0}=1.5$ as a consequence of the turbine rotor's flow blockage. 


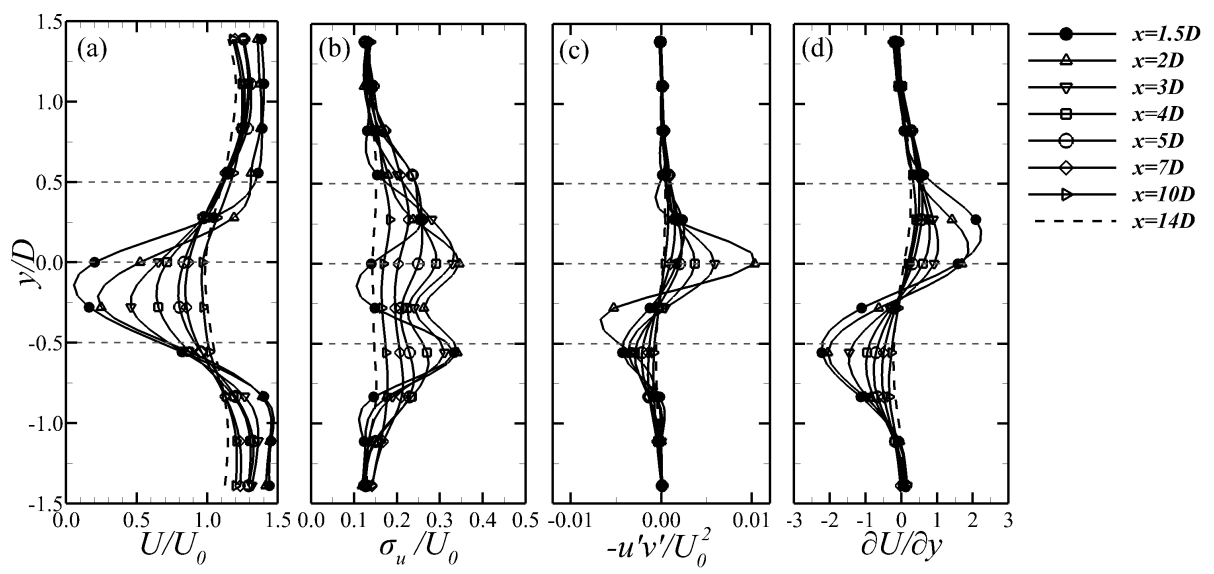

Figure 5: Horizonal profiles at rotor mid-height of (a) streamwise velocity, (b) streamwise velocity fluctuations, (c) shear stress $-u^{\prime} v^{\prime}$, and (d) horizontal gradient of mean velocity $\partial U / \partial y$.

In accordance with the distribution of $U$ in Fig. 4 a, the velocity asymmetry reduces after $x / D=7-10$ with minima of $U$ above $0.8 U_{0}$.

The profiles of $\sigma_{u}$ in Fig. $4 \mathrm{~b}$ exhibit two distinct distributions. In the near wake (at $x / D=1.5$ ) a distinct low-turbulence region is identified immediately downstream of the rotor, it coincides with low-momentum and is the result of the blades blocking the flow. The two peaks in the $x / D=1.5 \sigma_{u}$ profile signify the dynamic stall and/or trailing vortices, respectively, which are being flushed out of the inner turbine area. In the mid-wake two distinct peaks located at $y / D=0.0$ and -0.5 coinciding with regions of significant turbulence as seen in Fig. 4 are seen and, these are the signatures of aforementioned vortices being advected downstream. The peaks diminish further away from the turbine rotor suggesting that the vortices weaken as the wake is being filled with ambient fluid. Between $x / D=2$ and 5 , previously elevated levels of streamwise turbulence in the bypass flow reduce to those found in free-stream conditions. From $x / D=7$ onwards, the profiles of $\sigma_{u}$ smooth-out across the channel width until a uniform value of $\sigma_{u} / U_{0}=0.12$ is attained at $x / D=14$.

The location of the largest momentum exchange between the turbine wake and the free-stream flow is identified by maxima and minima in the $-u^{\prime} v^{\prime}$ profiles 
presented in Fig. 5F. Positive peaks of the transversal shear stress are found at approximately $y / D=0$ while negative peaks are found at $y / D=-0.5$ at most locations except for $x / D=2$. Momentum exchange is a result of the streamwise velocity gradients, $\partial U / \partial y$, and Fig. 5 d presents spanwise profiles of $\partial U / \partial y$. As can be seen their distribution is similar to $-u^{\prime} v^{\prime}$, except in the near-wake which is dominated by flow overtopping and undergoing the turbine rotor and hence at the location of the peak of the velocity gradient at $x / D=1.5$ there is relatively low spanwise shear.

\subsection{Vertical longitudinal plane}

The longitudinal plane along the vertical direction through the centre of the channel is presented in Fig. 6 showing contours of $U / U_{0}, \sigma_{u} / U_{0}, k / U_{0}^{2}$ and $-u^{\prime} w^{\prime} / U_{0}^{2}$. The low-momentum area extends over the entire projected rotor swept area, i.e. $-0.4<z / D<0.45$ and vanishes after $x / D \approx 1.8$. A certain asymmetry of the wake is observed in the vertical direction, evident from the iso-line of $U / U_{0}=0.25$ which is skewed towards the bottom half of the channel and extending until $x / D \approx 2$. This is caused by the downwash of fluid due to the flow overtopping the rotor depicted from the flow streamlines in Fig. 6b, which aids in recovering wake momentum mainly above $z / D=0$. An upwards motion arises from the gap between the rotor's bottom tips and the channel bed which enhances the wake recovery for $z / D<0$ but this is less intense than the overtopping flow. The difference in wake recovery rate above and beneath the turbine rotor is similar to the behaviour of the wake behind vertical axis wind turbines operating in a boundary layer flow [12].

In the transitional-wake region, i.e. $2<x / D<5$, the interaction between the fast-moving water above the rotor and the low-momentum wake area triggers high levels of turbulence as depicted in Fig. 6b and c. Contours of the Reynolds shear stresses $-u^{\prime} w^{\prime} / U_{0}^{2}$ are plotted in Fig. 6d showing an axi-symmetric distribution with respect to $z / D=0$. It can further be seen that this shear stress converges to a fairly uniform distribution over the water depth once the far-wake region is reached, i.e. $x / D>5$. 


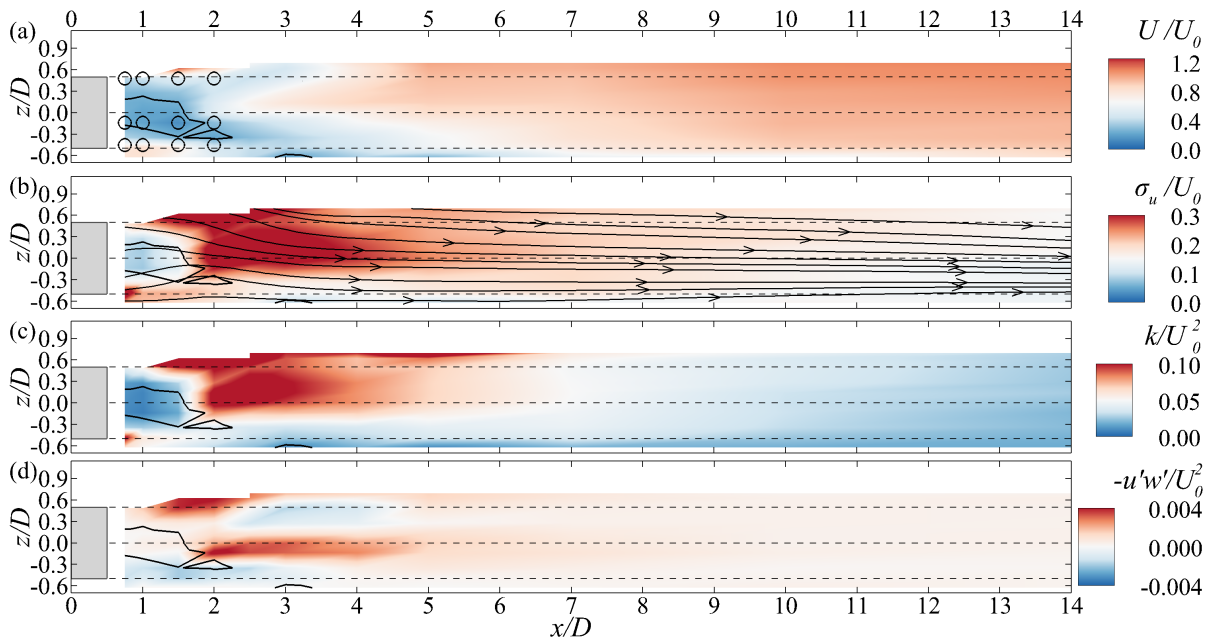

Figure 6: Vertical plane at $y / D=0$ with distribution of (a) streamwise velocity, (b) streamwise velocity fluctuation, (c) turbulent kinetic energy and (d) Reynolds shear stress $-u^{\prime} w^{\prime}$. Solid line indicates $U=0.25 U_{0}$.

Figure 7 presents profiles of $U, \sigma_{u},-u^{\prime} w^{\prime}$ and $\partial U / \partial z$ at different crosssections downstream of the rotor. The vertical profiles of $U$ exhibit a clear nonuniformity in the wake recovery behind the turbine, especially in the locations closest to the turbine at $x / D>3$. The different velocity magnitude between the flow overtopping the rotor and that coming from the bottom part of the channel induce an uneven vertical distribution of the streamwise velocity in the profiles along the mid-wake. Analogously to the horizontal profiles in Fig. 5 , streamwise momentum is almost recovered at $x / D=10$ as its distribution matches that at $x / D=14$ being both very close to the free-stream profile from Fig. 11.

The profile at $x / D=1.5$ intersects the low-momentum region and the vertical profile of $\sigma_{u}$ exhibits values in the range of $0.12-0.15$, i.e. close to the free-stream turbulence intensity and hence as mentioned suggests that the lowmomentum area features very low turbulence intensities as well. Downstream of the near wake the mixing between the turbine's wake and ambient flow increases and streamwise turbulence intensity reaches a peak of approx. 0.35 at $x / D=2$ and 3 in between $-0.45<z / D<0.4$. Note that near the free-surface at 1.5 

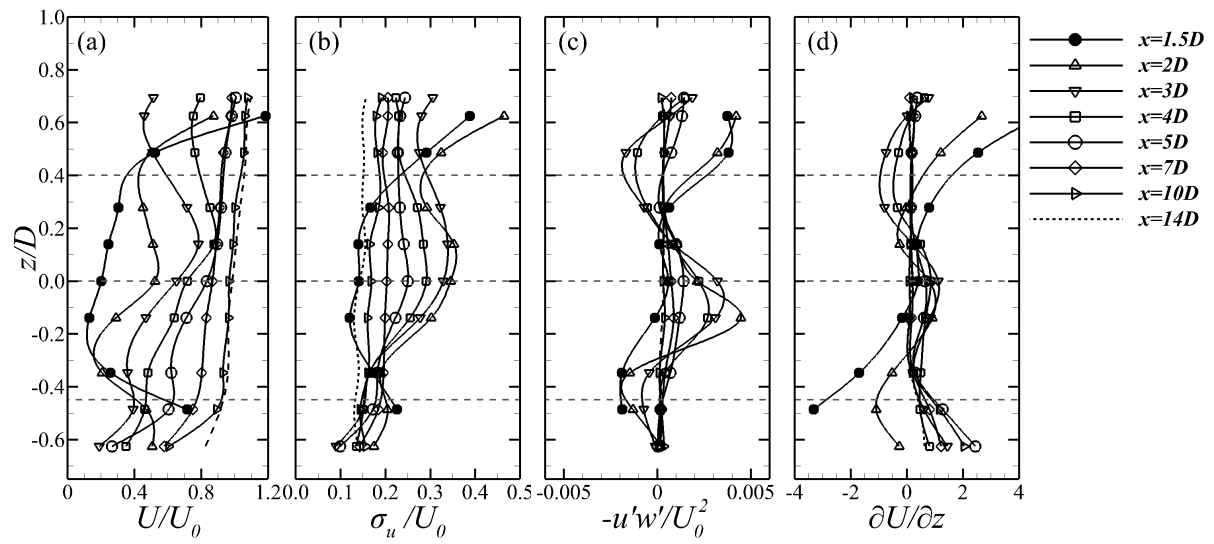

Figure 7: Vertical profiles at flume centre of (a) streamwise velocity, (b) streamwise velocity fluctuations (c) Reynolds shear stress $-u^{\prime} w^{\prime}$, and (d) vertical gradient of streamwise velocity, $\partial U / \partial z$.

and 2 diameters downstream the turbine rotor, $\sigma_{u}$ is triggered as a result of the turbulent downwash, i.e. flow coming from the top of the rotor. After $x / D=3$ there is a progressive decay of $\sigma_{u}$ that features the highest values in the region $-0.2<z / D<0.4$. In the far-wake region, $\sigma_{u}$ exhibits a similar value to those found in free-stream flow conditions.

The mid-wake exhibits a notably irregular distribution of $-u^{\prime} w^{\prime}$ along the water depth as a result of the complex interaction between the turbine wake with the ambient flow whilst in the far-wake the distribution of this shear stress is found to be more uniform with negligible values beyond $x / D=10$. In Fig. $7 \mathrm{p}$ the vertical gradient of mean streamwise velocity is plotted and its distribution is somewhat similar to that of $-u^{\prime} w^{\prime}$.

The Boussinesq approximation for isotropic turbulent flow states that Reynolds shear stresses are proportional to the velocity gradients, i.e. $-u^{\prime} v^{\prime}=\nu_{t} \frac{\partial U}{\partial y}$ and $-u^{\prime} w^{\prime}=\nu_{t} \frac{\partial U}{\partial z}$ where $\nu_{t}$ denotes eddy viscosity. In the present flow such an approximation would not be valid in the near- and mid-wake regions as the profiles of shear stress and velocity gradients do not show peaks at the same location suggesting that the eddy viscosity would have to vary quite significantly over the depth. 


\subsection{Cross-sectional planes}

The three-dimensional nature of the turbine wake is further investigated in Fig. 8 showing the distribution of streamwise velocity, streamwise velocity fluctuation and shear stresses in four cross-sectional planes (i.e. at $x / D=1,2$, 3 and 5) normal to the flow direction.

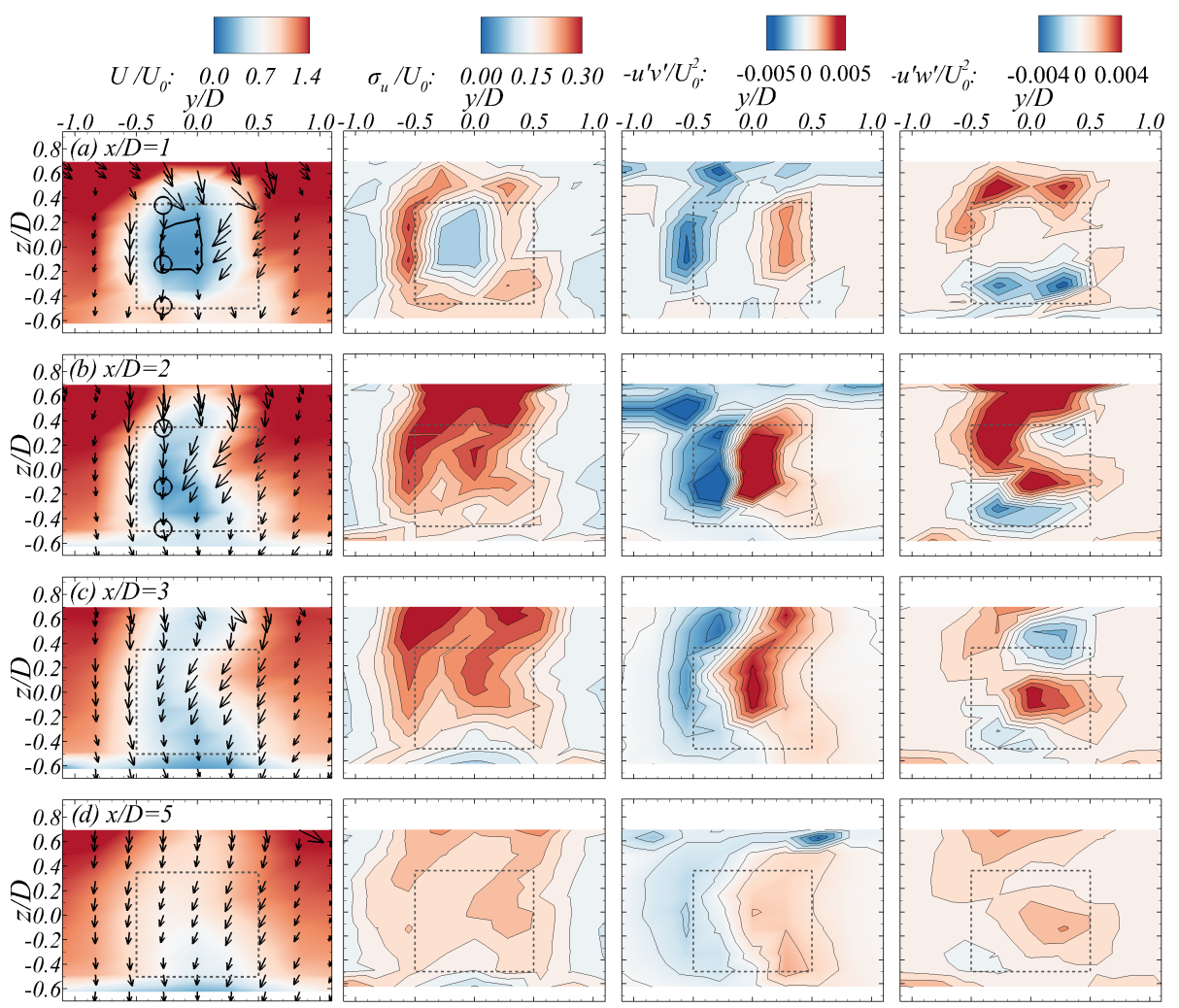

Figure 8: Distribution of normalised streamwise velocity $\left(U / U_{0}\right)$, turbulence intensity $\left(\sigma_{u} / U_{0}\right)$ and Reynolds shear stresses $\left(-u^{\prime} v^{\prime}\right.$ and $\left.-u^{\prime} w^{\prime}\right)$ along four cross-sections downstream of the turbine at $x / D=1,2,3$ and 5 . Solid line indicates $U=0.25 U_{0}$.

285

At the section closest to the turbine $(x / D=1)$, the region of $U<0.25 U_{0}$ (solid lines) is predominantly found for $y / D<0$. Velocity vectors illustrate that in all considered sections the ambient flow entrains into the wake region more pronouncedly for $y / D>0$ with large negative vertical velocities as a result 290

of the downwash. At $x / D=1$, the regions of high turbulence levels coincide with the projected rotor's swept perimeter which is in agreement with the path 
described by the dynamic stall vortices shown in Fig. 4. The largest values of shear stresses are found along $y / D=-0.6$ and 0.4 for $-u^{\prime} v^{\prime}$ and $z / D=-0.35$ and 0.55 for $-u^{\prime} w^{\prime}$. The peaks of $-u^{\prime} w^{\prime}$ almost coincide with the projected location of the blade's tips as they are slightly shifted upwards.

At two diameters downstream of the turbine, the low-momentum region expands over a larger vertical area and velocity minima surpass values above $0.25 U_{0}$. Velocity vectors evidence the inwards interaction of the bypass flow into the low-velocity area generating a "C"-shaped distribution of the wake. The largest values of $\sigma_{u}$ are observed between the free-surface and top of the rotor as a result of the overtopping flow, and also at $y / D \approx-0.5$ and 0.0 where the change from low to high streamwise velocities is relatively fast, as depicted form the $\partial U / \partial y$ profile in Fig. 5d. The highest $-u^{\prime} v^{\prime}$ and $-u^{\prime} w^{\prime}$ values are located within the rotor swept area as a result of the larger entrainment of high-momentum flow from the ambient flow into the low-momentum wake. At $x / D=3$ and 4 (the latter not shown here), a similar distribution of $U, \sigma_{u}$ and $-u^{\prime} v^{\prime}$ to that at $x / D=2$ is found as these all belong to the transitional-wake region in which the wake maintains a similar pattern. Further downstream at $x / D>5$, the dynamics of the wake changes as the momentum is recovered and the "C"-shaped low-velocity region is no longer observed. The magnitudes of the Reynolds stresses are also reduced whilst their overall distribution remains similar to that found in the cross-sections at $x / D=2$ and 3 .

\subsection{Power spectra}

The transition from near-wake to transitional-wake is marked by the role of the dynamic stall vortices in the momentum recovery. The presence of these large-scale turbulent structures are identified in Fig. 9 via Power Spectral Density (PSD) of u-velocity time-series presented. In the calculation of the PSDs, each signal was decomposed into 10 overlapping segments spanning 60 s each and a Hann window was applied in order to obtain each of their transformations. The resulting spectra are the average of these segments.

A total of 12 PSDs are computed from points vertically aligned with the 


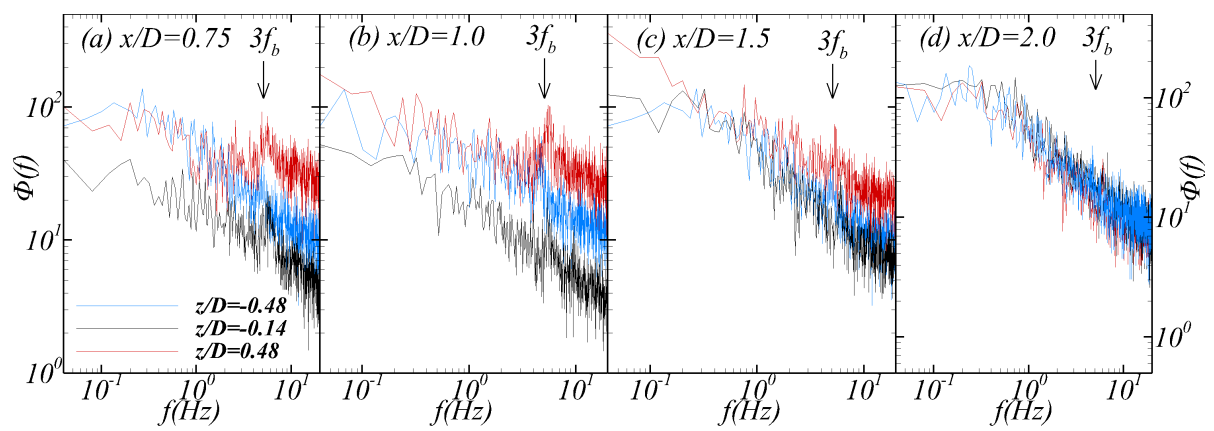

Figure 9: Power spectral density, $\Phi$, of u-velocity obtained at three vertical locations at four locations downstream of the turbine.

top, centre and bottom of the wake at different locations within the near-wake as indicated in Fig. 6a. At distances of $x / D=0.75$ and 1.0 the spectra show a clear energetic peak at a frequency of $3 f_{b}$, with $f_{b}$ standing for the blade passing frequency, revealing the presence of dynamic stall vortices shed by the turbine rotor blades. At $x / D=1.5$, the energy peaks become more diffused as a result of the energetic vortices losing coherence during their convection downstream while interacting with the turbulent ambient flow. In the onset of the midwake region at $x / D=2.0$, no significant energy peaks at a frequency of $3 f_{b}$ are discerned indicating that dynamic stall vortices are no longer coherence at this stage of the wake, which allows a larger entrainment of the bypass flow into the low-velocity area of the wake.

\subsection{Streamwise momentum recovery}

The contributors to the replenishment of the momentum deficit behind the turbine as a result of its interaction with the main flow are accounted via the Reynolds-averaged Navier-Stokes (RANS) equation in the x-direction, which reads,

$$
\frac{\partial U}{\partial x}=\frac{1}{U}(\underbrace{-V \frac{\partial U}{\partial y}}_{\mathrm{I}} \underbrace{-W \frac{\partial U}{\partial z}}_{\mathrm{II}} \underbrace{-\frac{\partial u^{\prime} u^{\prime}}{\partial x}}_{\mathrm{III}} \underbrace{-\frac{\partial u^{\prime} v^{\prime}}{\partial y}}_{\mathrm{IV}} \underbrace{-\frac{\partial u^{\prime} w^{\prime}}{\partial z}}_{\mathrm{V}} \underbrace{-\frac{1}{\rho} \frac{\partial P}{\partial x}}_{\mathrm{VI}} \underbrace{+\nu \frac{\partial^{2} U}{\partial x_{i}^{2}}}_{\mathrm{VII}})
$$


In the present analysis, the flow is deemed stationary in its time-averaged

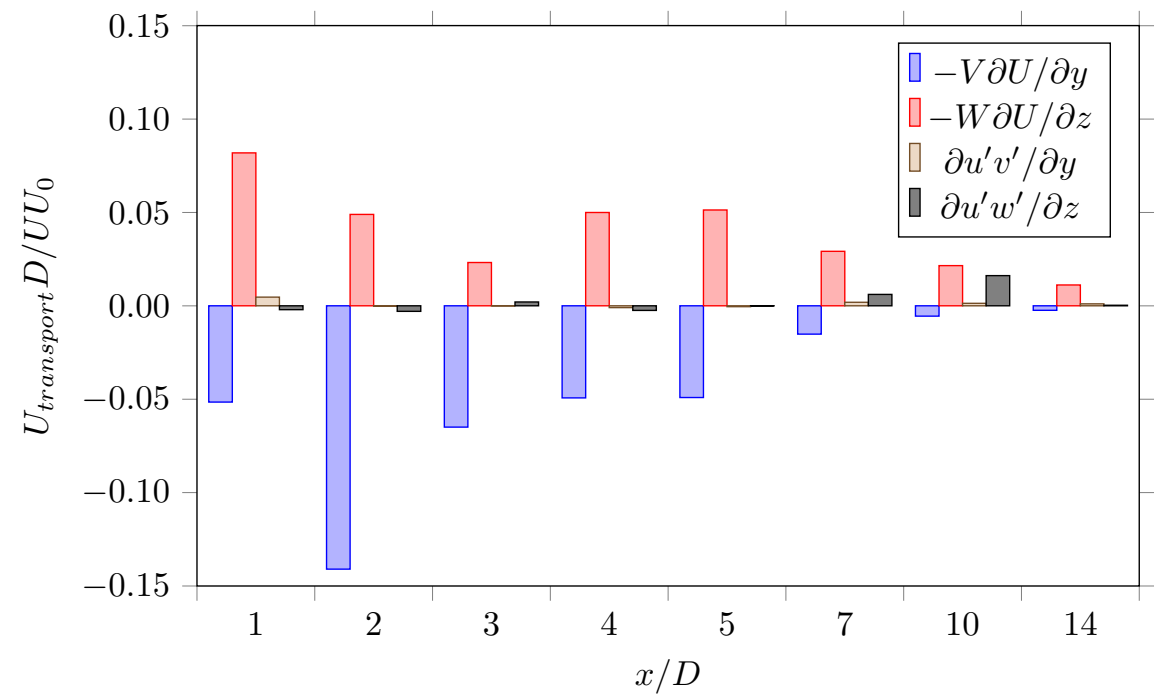

Figure 10: Cross-sectional average values of the streamwise momentum equation contributors along the locations measured.

Comparing the relative contribution between the advection terms at $x / D=$ 1 , vertical advection has a greater value over the transversal advection suggesting that in the near wake, overtopping and underflow of the turbine rotor are 
mainly responsible for filling the turbine wake and this is in line with the findings of Bachant and Wosnik [8]. At $x / D=2$, the lateral flow entrainment (i.e. $\mathrm{y}$-advection) reaches a peak and it is approximately three times greater than the $\mathrm{z}$-advection term. Within the region between $x / D=3-5$, the momentum deficit recovers and this is evidenced with a decrease in the magnitudes of spanwise advection reaching similar values to vertical advection. In the far-wake, the values of the advection terms diminish whilst the turbulent transport terms seem to increase at $x / D=7$ and $x / D=10$.

These results indicate that close to the rotor vertical advection is the key ingredient to recover momentum in the wake, while in the transition from nearto mid-wake spanwise advection dominates. Along the mid-wake and over the far-wake, both advection terms have a similar net contribution, which results from the large mixing of the rotor-induced wake with the ambient flow.

\subsection{Skewness of the turbine rotor's wake}

Skewness, or third-order moment, indicates the asymmetry of the probability density function of velocities. Chamorro et al. [20] outlined the importance of flow skewness together with mean velocity and turbulence intensity to determine the available power that can be harnessed by wind turbines. Therefore, it is of particular interest to quantify it in the wake downstream of the vertical axis turbine as it can provide further insights into high-order wake effects towards designing future arrays of turbines. The skewness of the $i$-component of velocity fluctuations $S k_{u_{i}}$ is defined as:

$$
S k_{u_{i}}=\frac{\sum_{n=1}^{N} u_{i}^{\prime}\left(t_{n}\right)^{3}}{N \sigma_{u_{i}}^{3}}
$$

Figure 11 presents contours of the streamwise $\left(S k_{u}\right)$ and spanwise $\left(S k_{v}\right)$ skewness together with iso-lines of the streamwise velocity at four different locations in the transitional- and far-wake region, namely $x / D=3,4,7$ and 10. At $x / D=3$, areas of large positive $S k_{u}$ correlate with low streamwise ve- 
locities and vice versa, whilst areas of approximately zero skewness are found where the spanwise shear stress, $-u^{\prime} v^{\prime}$ is maximum. Velocity arrows in Fig. 8. indicate the inwards motion of the free-stream flow into the low-momentum region at $y / D>0$ resulting in negative values of $S k_{v}$. The distribution of the skewness of $\mathrm{u}$ - and v-velocities at $x / D=4$ is quite similar to those observed at $x / D=3$ although $S k_{v}$ features larger values at the latter location due to a more pronounced interaction between the turbine's wake and the free-stream flow. The pattern of $S k_{u}$ and $S k_{v}$ at $x / D=7$ and 10 are comparable although with smaller values to those found at $x / D=3$ or $x / D=4$, showing that rotorinduced effects in the flow field remain present until $10 D$ downstream of the device. Skewness contours at $14 D$ (not shown here for brevity) show a similar distribution to those found in free-stream conditions.
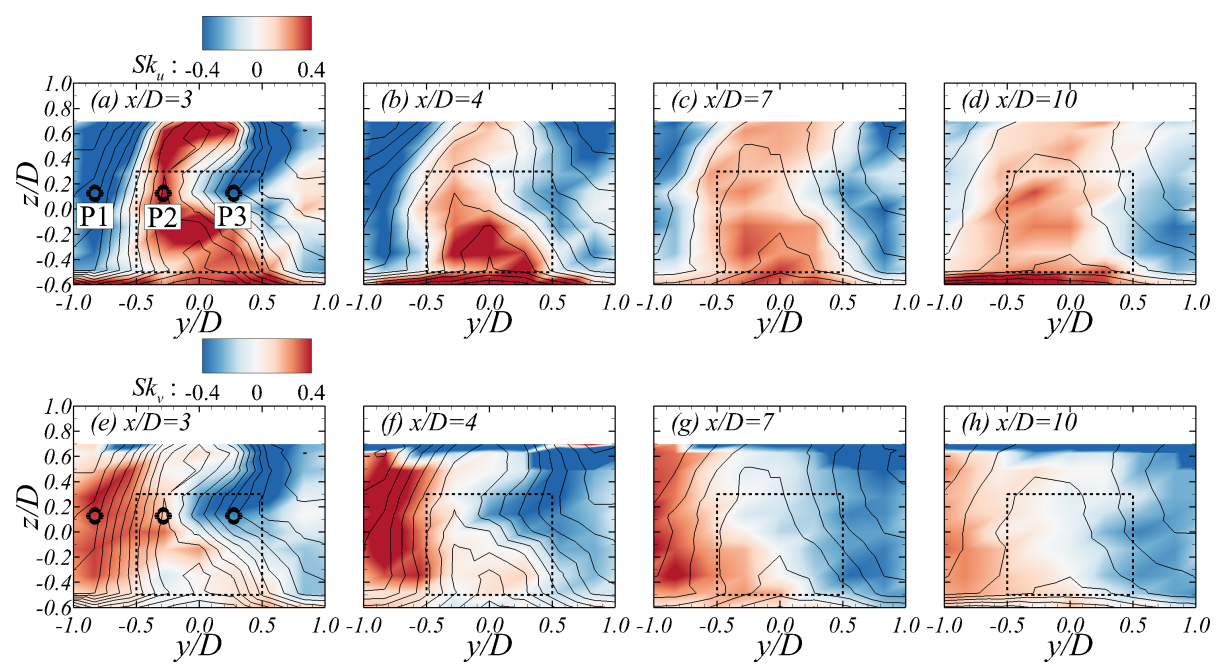

Figure 11: Contours of (a-d) $S k_{u}$ and (e-h) $S k_{v}$ at different cross-sections behind the turbine.

The velocity fluctuations at $x / D=3$ are further analysed at three selected locations P1, P2 and P3 (indicated in Fig. 11a and e) which belong to regions with distinct velocity skewness. Probability Density Functions (PDFs) of $u^{\prime}$ and $v^{\prime}$ at those locations are shown in Fig. 12 to c. The PDFs at P1 indicate a reversed skewed distribution of $u^{\prime}$ compared to $v^{\prime}$ being the median of $u^{\prime}$ positive while that of $v^{\prime}$ negative, albeit both of similar magnitude. The PDF 
of $u^{\prime}$ features a longer tail along the negative values until $u^{\prime} / \sigma_{u}<-4$ whilst in the positive region of $S k_{u}$ it reaches almost zero values for $u^{\prime} / \sigma_{u}>2$. Such distribution indicate that on the negative side there are fewer turbulent events but these have a larger turbulence intensity that events triggering positive $u^{\prime}$ which happen more frequently and with lower intensity. At the centre of the wake, i.e. at P2, both PDFs exhibit an almost Gaussian distribution. On the other side of the wake, at $\mathrm{P} 3$, the PDFs of $u^{\prime}$ and $v^{\prime}$ are biased to the positive side with a similar positive median which is again in good correlation with contours of skewness (Fig. 11 and e) as these are both of negative and of similar magnitude at this point.
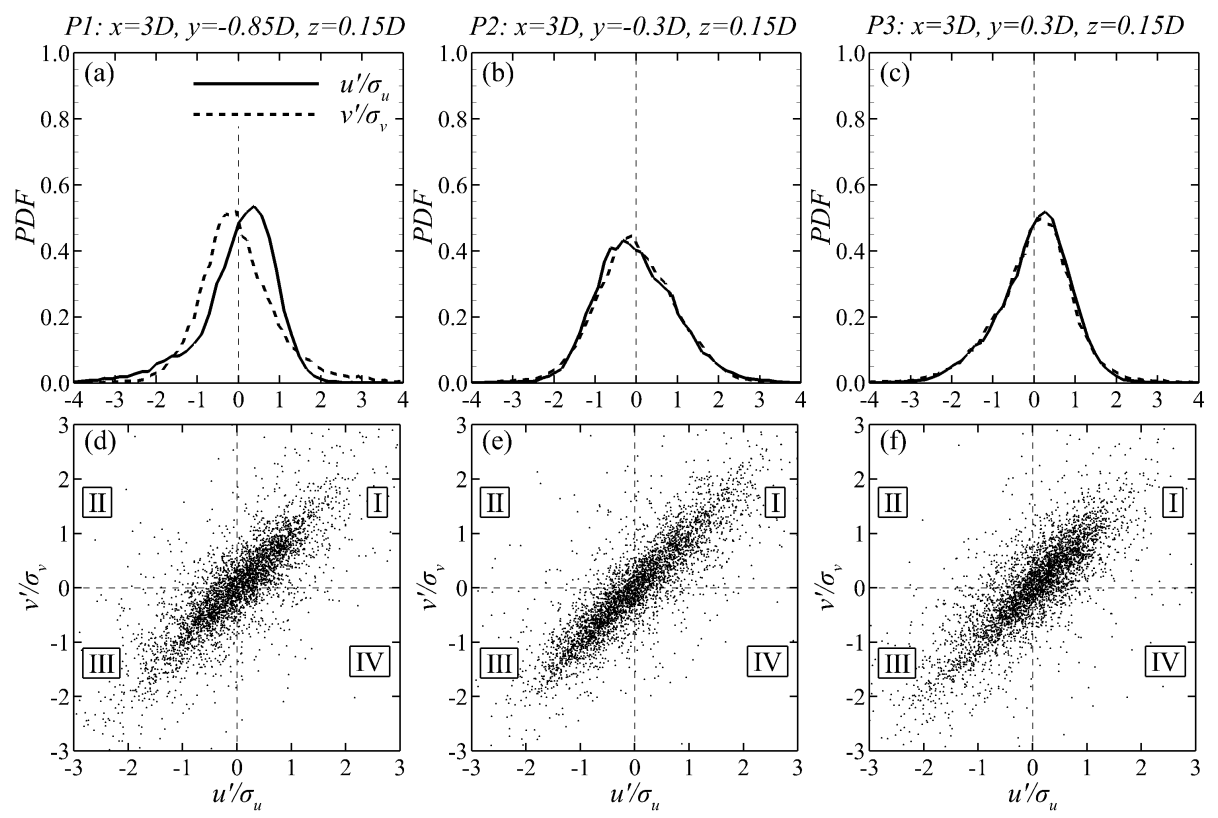

Figure 12: Probability density functions (top row) and quadrant analysis (bottom row) of velocity fluctuations at $\mathrm{P} 1, \mathrm{P} 2$ and $\mathrm{P} 3$.

These observations on the asymmetric distribution of velocity fluctuations are complemented with Figs. $12 \mathrm{~d}$ to f which plot normalised values of velocity fluctuations in streamwise and spanwise directions. The quadrant analysis at the three reference points show that the majority of the velocity fluctuation values reside within the quadrants I, i.e. $u^{\prime}>0, v^{\prime}>0$, and III, i.e. $u^{\prime}<0$, 
$v^{\prime}<0$. In the centre of the wake (P2) the symmetry in both PDFs agree with the quadrant analysis whose centre of mass is located at the origin of coordinates. The predominant distribution of velocity fluctuations over quadrants I and III suggests there is predominant outward and inward interactions and reflects the anisotropic nature of turbulence in the VAT's wake.

\subsection{Wake characteristics and recovery}

The recovery of the flow along the channel centreline is presented in Fig. 13. in terms of the three components of time-averaged velocity $\left(U_{i}\right)$ and velocity fluctuation $\left(\sigma_{u_{i}}\right)$, the velocity deficit in the streamwise direction $(\Delta U=(U-$ $\left.U_{0}\right)$ ) and the three Reynolds shear stresses $-u^{\prime} v^{\prime},-u^{\prime} w^{\prime}$ and $-v^{\prime} w^{\prime}$.
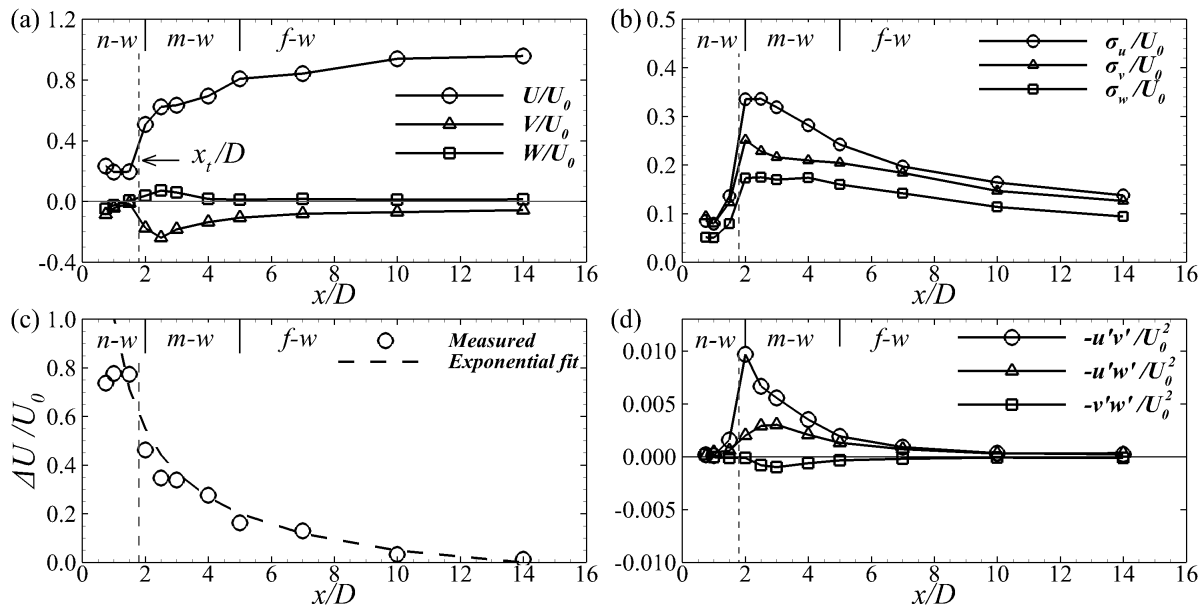

Figure 13: Distribution along the wake centreline of (a) normalised time-averaged velocity components, (b) turbulence intensities, (c) measured velocity deficit and exponential fit, and (d) Reynolds shear stresses. $n-w, m-w$ and $f-w$ denote near-, mid- and far-wake, respectively.

Figure 13 shows that the lowest streamwise velocites are found in the nearwake $(\mathrm{n}-\mathrm{w})$ with the minimum velocity $U_{\min }$ approximately equal to $0.2 U_{0}$ (which equates to a velocity deficit of $\Delta U / U_{0}=0.8$ ) located in the transition from near- to mid-wake $(\mathrm{m}-\mathrm{w})$ at about 2 diameters downstream of the rotor's shaft. Based on their measurements of the wake behind multiple VATs, Araya et al. 11] proposed that the location of the near-wake to medium-wake 
transition, $x_{t}$, can be approximated by,

$$
x_{t} / D=4.78-4.93 \sigma_{D}
$$

where $\sigma_{D}$ is the dynamic solidity, a parameter that links the design solidity of the turbine rotor $(\sigma, \mathrm{Eq} .4)$ with its actual tip speed ratio $\lambda$, and is calculated as,

$$
\sigma_{D}=1-\frac{1}{2 \pi \sigma \lambda}
$$

For the current study $\sigma_{D}$ is equal to 0.60 and thus applying Eq. 9 the value of $x_{t}$ is equal to $1.82 D$ which matches well the present observations. Considering the Reynolds number of this flow is almost one order of magnitude above that of Araya et al. [11]'s experiments, this match indicates that this equation is a fair estimate of the transition from near- to mid-wake for vertical axis turbines and not very sensitive to the Reynolds number of the flow.

Second order statistics also reach their minima in the near-wake region as shown in Fig. $13 \mathrm{~b}$ and $d$. In the onset of the mid-wake, the entrainment of external turbulent flow into the wake increases momentum but also triggers turbulence and thus velocity fluctuations attain their maxima. In other words, there is a fast transition from a quasi-steady region shortly behind the turbine $(\mathrm{n}-\mathrm{w})$ to a stage of fast-rate momentum recovery and high-turbulence levels $(\mathrm{m}-\mathrm{w})$. Transversal and vertical velocities feature their largest values in the ${ }_{425}$ mid-wake reaching a maximum of $V \approx-0.25 U_{0}$ and $W \approx 0.10 U_{0}$.

A more subtle transition of flow quantities is observed from mid- to farwake (f-w). Here, the streamwise velocity features a value of $U=0.8 U_{0}$ and achieves a magnitude close to the free-stream velocity at $x / D=10$. Vertical and transversal velocities as well as Reynolds stresses exhibit a progressive decay along the far-wake reaching their minima at $x / D=14$ and at $x / D=10$ for the shear stresses.

Albeit the complexity of the wake recovery pattern, the normalised stream- 
wise velocity deficit $\left(\Delta U / U_{0}\right)$ along the wake centreline is in agreement with the following distribution:

$$
\Delta U / U_{0}=a\left(\frac{x}{D}\right)^{-b}-U_{\min }
$$

in which $a$ and $b$ are 1.168 and 0.684 according to Araya et al. 11] who firstly proposed such exponential decay of the velocity deficit for VATs. Note this Eq. 11 allows to estimate the wake deficit recovery with just measuring $U_{\min }$ which is found in the near wake. Fig. 13 shows the velocity deficit attains its maximum value before the transition point $x_{t}$, and that beyond this location there is a good match between the measured velocity deficit and the exponential fit is fairly good.

\section{Discussion and conclusions}

The wake generated behind a vertical axis turbine operating at its peak efficiency has been characterised based on experiments carried out in Cardiff University's hydraulics laboratory. The three-dimensional nature of the wake has been analysed using ADV and the characteristics of the turbine rotor's wake have been identified.

${ }_{445}$ The wake can be divided into three distinct regions: (i) a near-wake region featuring a low-momentum area just downstream of the turbine rotor in which dynamic stall vortices play a key role and limit the entrainment of ambient flow into the low-velocity region; (ii) a transitional-wake region, extending 2 to 5 diameters downstream of the turbine rotor that is characterised by a fastrate of momentum recovery and high intensity of turbulence and, in addition, a C-shaped distribution of the low-velocities due to the inwards motion of the ambient flow entraining into the wake generating an asymmetric distribution of the velocity field; and (iii) a far-wake that begins at approximately 5 diameters downstream of the rotor with an almost full recovery of the momentum deficit as streamwise velocities reach values above 0.8 times the bulk velocity, whilst high-order effects, such as elevated levels of flow skewness in comparison to 
those in free-stream conditions, persist until a distance of 14 rotor diameters downstream of the turbine location.

The contours of flow skewness and the probability distribution function at selected locations evidenced the effect of the rotating rotor in producing an asymmetric wake in terms of flow velocities and turbulence. The quadrant analysis of the streamwise and spanwise velocity fluctuations suggested strong inwards and outwards interactions again the result of flow asymmetry. The integration of the streamwise Reynolds-averaged momentum equation quantified that transversal and vertical advection terms are main contributors to momentum replenishment. In the near wake vertical advection is the most relevant, due to significant flow overtopping and undergoing the rotor, while spanwise advection is the main contributor to momentum replenishment in the transitional wake. Despite the complex mechanisms involved in the wake recovery, the velocity deficit recovery can be approximated with reasonable accuracy with an empirical exponential distribution.

\section{Acknowledgements}

The authors acknowledge Cardiff University and EMRGY for partially sponsor this research.

[1] M. Esteban, D. Leary, Current developments and future prospects of offshore wind and ocean energy, Applied Energy 90 (1) (2012) 128-136. doi:10.1016/j.apenergy.2011.06.011.

[2] P. Bachant, M. Wosnik, Effects of Reynolds Number on the Energy Conversion and Near-Wake Dynamics of a High Solidity Vertical-Axis Cross-Flow Turbine, Energies 9 (2) (2016) 73. doi:10.3390/en9020073.

[3] C. Simão Ferreira, G. Van Kuik, G. van Bussel, F. Scarano, Visualization by PIV of dynamic stall on a vertical axis wind turbine, Experiments in Fluids 46 (1) (2009) 97-108. doi:10.1007/s00348-008-0543-z. 
[4] P. Ouro, T. Stoesser, An immersed boundary-based large-eddy simulation approach to predict the performance of vertical axis tidal turbines, Computers \& Fluids 152 (2017) 74-87. doi:10.1016/j.compfluid.2017.04.003

[5] G. Brochier, P. Fraunie, C. Beguier, I. Paraschivoiu, Water channel experiments of dynamic stall on Darrieus wind turbine blades, Journal of Propulsion and Power 2 (5) (1986) 445-449. doi:10.2514/3.22927.

[6] M. Somoano, F. Huera-Huarte, Flow dynamics inside the rotor of a three straight bladed cross-flow turbine, Applied Ocean Research 69 (2017) 138147. doi:10.1016/j.apor.2017.10.007.

[7] G. Tescione, D. Ragni, C. He, C. J. Simão Ferreira, G. van Bussel, Near wake flow analysis of a vertical axis wind turbine by stereoscopic particle

495 image velocimetry, Renewable Energy 70 (2014) 47-61. doi:10.1016/j. renene.2014.02.042

[8] P. Bachant, M. Wosnik, Performance measurements of cylindrical- and spherical-helical cross-flow marine hydrokinetic turbines, with estimates [ of exergy efficiency, Renewable Energy 74 (2015) 318-325. doi:10.1016/ j.renene.2014.07.049.

[9] M. Kinzel, Q. Mulligan, J. O. Dabiri, Energy exchange in an array of 1. vertical-axis wind turbines, Journal of Turbulence 14 (6) (2012) N38. doi: $10.1080 / 14685248.2012 .712698$.

[10] P. Ouro, T. Stoesser, Wake Generated Downstream of a Vertical Axis Tidal Turbine, in: 12th European Wave and Tidal Energy Conference (EWTEC), Cork, Ireland, 2017.

[11] D. B. Araya, T. Colonius, J. O. Dabiri, Transition to bluff-body dynamics in the wake of vertical-axis wind turbines, Journal of Fluid Mechanics 813 (2017) 346-381. doi:10.1017/jfm.2016.862. 
[12] V. F.-C. Rolin, F. Porté-Agel, Experimental investigation of vertical-axis wind-turbine wakes in boundary layer flow, Renewable Energy 118 (2018) 1-13. doi:10.1016/j.renene.2017.10.105

[13] K. J. Ryan, F. Coletti, C. J. Elkins, J. O. Dabiri, J. K. Eaton, Threedimensional flow field around and downstream of a subscale model rotating vertical axis wind turbine, Experiments in Fluids 57 (3) (2016) 1-15. doi: $10.1007 / \mathrm{s} 00348-016-2122-z$

[14] T. Harries, A. Kwan, J. Brammer, R. Falconer, Physical testing of performance characteristics of a novel drag-driven vertical axis tidal stream turbine; with comparisons to a conventional Savonius, International Journal of Marine Energy 14 (2016) 215-228. doi:10.1016/j.ijome.2016.01.008.

[15] L. Priegue, T. Stoesser, The influence of blade roughness on the performance of a vertical axis tidal turbine, International Journal of Marine Energy 17 (2017) 136-146. doi:10.1016/j.ijome.2017.01.009

[16] P. Ouro, M. Harrold, T. Stoesser, P. Bromley, Hydrodynamic loadings on a horizontal axis tidal turbine prototype, Journal of Fluids and Structures 71 (2017) 78-95. doi:10.1016/j.jfluidstructs.2017.03.009.

[17] L. Cea, J. Puertas, L. Pena, Velocity measurements on highly turbulent free surface flow using ADV, Experiments in Fluids 42 (3) (2007) 333-348. doi:10.1007/s00348-006-0237-3.

[18] I. Nezu, H. Nakagawa, Turbulence in open-channel flows, Rotterdam, The Netherlands: A. A. Balkema, 1993.

[19] D. B. Araya, J. O. Dabiri, A comparison of wake measurements in motordriven and flow-driven turbine experiments, Experiments in Fluids 56 (2015) 150. doi:10.1007/s00348-015-2022-7.

[20] L. Chamorro, M. Guala, On the evolution of turbulent scales in the wake 口 of a wind turbine model, Journal of Turbulence 13 (27) (2012) 1-13. doi: $10.1080 / 14685248.2012 .697169$. 